Proyecciones Journal of Mathematics

Vol. 36, No 4, pp. 545-566, December 2017.

Universidad Católica del Norte

Antofagasta - Chile

\title{
The generalized Van Vleck's equation on locally compact groups
}

\author{
B. Fadli \\ IBN Tofail University, Morocco \\ D. Zeglami \\ Moulay Ismail University, Morocco and \\ S. Kabbaj \\ IBN Tofail University, Morocco \\ Received : November 2016. Accepted : April 2017
}

\begin{abstract}
We determine the continuous solutions $f, g: G \rightarrow \mathbf{C}$ of each of the two functional equations

$$
\begin{aligned}
& \int_{G}\{f(x y t)-f(\sigma(y) x t)\} d \mu(t)=f(x) g(y), \quad x, y \in G, \\
& \int_{G}\{f(x y t)-f(\sigma(y) x t)\} d \mu(t)=g(x) f(y), \quad x, y \in G,
\end{aligned}
$$

where $G$ is a locally compact group, $\sigma$ is a continuous involutive automorphism on $G$, and $\mu$ is a compactly supported, complex-valued Borel measure on $G$.
\end{abstract}

Subjclass [2010] : Primary 39B32, 39B52.

Keywords : Functional equation, Van Vleck, involutive automorphism, character, additive map. 


\section{Introduction}

In the papers [8,9], Van Vleck studied the continuous solutions $f: \mathbf{R} \rightarrow \mathbf{R}$, $f \neq 0$, of the functional equation

$$
f\left(x-y+z_{0}\right)-f\left(x+y+z_{0}\right)=2 f(x) f(y), \quad x, y \in \mathbf{R},
$$

where $z_{0}>0$ is fixed. We shall in this paper study extensions of (1.1) and related functional equations from $\mathbf{R}$ to locally compact groups.

Let $G$ be a group and $Z(G)$ be the center of $G$. In [4], Perkins and Sahoo extended the result of Van Vleck by determining the abelian solutions $f: G \rightarrow \mathbf{C}$ of the functional equation

$$
f\left(x \tau(y) z_{0}\right)-f\left(x y z_{0}\right)=2 f(x) f(y), \quad x, y \in G,
$$

where $z_{0} \in Z(G)$ and $\tau: G \rightarrow G$ is an involution (that is, an antihomomorphism such that $\tau(\tau(x))=x$ for all $x \in G$ ). As a very recent result, Stetkær extended the result of Perkins and Sahoo in [7] by solving the functional (1.2) on semigroups.

Van Vleck's functional equation (1.1) was generalized in another direction by the authors in [3], viz. to the functional equation

$$
f\left(\sigma(y) x z_{0}\right)-f\left(x y z_{0}\right)=2 f(x) f(y), \quad x, y \in G,
$$

where $z_{0} \in G$ is a fixed element that need not belongs to $Z(G)$ and $\sigma: G \rightarrow$ $G$ is an involutive automorphism (that is involutive means that $\sigma(\sigma(x))=x$ for all $x \in G$ ). Observe that (1.3) can be written as follows

$$
\int_{G}\{f(\sigma(y) x t)-f(x y t)\} d \mu(t)=f(x) f(y), \quad x, y \in G,
$$

where $\mu=\frac{1}{2} \delta_{z_{0}}\left(\delta_{z_{0}}\right.$ is the Dirac measure concentrated at $\left.z_{0}\right)$. Our aim is to generalize this equation by substituting the Dirac measure by an arbitrary compactly supported, complex-valued Borel measure and considering more unknown functions.

Let $G$ be a locally compact group, $\sigma$ be a continuous involutive automorphism on $G$, and $\mu$ be a compactly supported, complex-valued Borel measure on $G$. The purpose of the present paper is first to give an explicit description of the continuous solutions $f, g: G \rightarrow \mathbf{C}$ of each of the two integral-functional equations 


$$
\begin{aligned}
& \int_{G}\{f(x y t)-f(\sigma(y) x t)\} d \mu(t)=f(x) g(y), \quad x, y \in G, \\
& \int_{G}\{f(x y t)-f(\sigma(y) x t)\} d \mu(t)=g(x) f(y), \quad x, y \in G,
\end{aligned}
$$

and second to present several consequences of these results as well. In particular, using our main results (Theorem 3.3 and Theorem 4.2), we find the continuous solutions $f: G \rightarrow \mathbf{C}$ of the following Van Vleck's integralfunctional equation

$$
\int_{G}\{f(\sigma(y) x t)-f(x y t)\} d \mu(t)=f(x) f(y), \quad x, y \in G,
$$

and the solutions $f, g: G \rightarrow \mathbf{C}$ of each of the equations

$$
\begin{gathered}
f\left(x y z_{0}\right)-f\left(\sigma(y) x z_{0}\right)=2 f(x) g(y), \quad x, y \in G, \\
f\left(x y z_{0}\right)-f\left(\sigma(y) x z_{0}\right)=2 g(x) f(y), \quad x, y \in G, \\
\sum_{i=0}^{n}\left\{f\left(x y z_{i}\right)-f\left(\sigma(y) x z_{i}\right)\right\}=2 f(x) g(y), \quad x, y \in G, \\
\sum_{i=0}^{n}\left\{f\left(x y z_{i}\right)-f\left(\sigma(y) x z_{i}\right)\right\}=2 g(x) f(y), \quad x, y \in G,
\end{gathered}
$$

where $z_{0}, z_{1}, \ldots, z_{n} \in G$ are fixed elements. Note that each of the equations (1.7)-(1.10) results from (1.4) or (1.5) by replacing $\mu$ by a suitable discrete measure and that all these equations are, according to our knowledge, not in the literature even for abelian groups.

Results of $[1,2,3,5,10]$ have been an inspiration by their treatments of similar functional equations on groups.

\section{Notation and terminology}

To formulate our results we recall the following notation and assumptions that will be used throughout the paper:

Let $G$ be a group with neutral element $e$. The map $\sigma: G \rightarrow G$ denote an involutive automorphism. That it is involutive means that $\sigma(\sigma(x))=x$ for all $x \in G$. If $(G,+)$ is an abelian group, then the inversion $\sigma(x):=-x$ is an 
example of an involutive automorphism. Another example is the complex conjugation map on the multiplicative group of non-zero complex numbers.

For any complex-valued function $F$ on $G$ we use the notations

$$
F_{e}=\frac{F+F \circ \sigma}{2} \quad \text { and } \quad F_{o}=\frac{F-F \circ \sigma}{2} .
$$

We say that $F$ is even if $F=F_{e}$, and odd if $F=F_{o}$.

A function $A: G \rightarrow \mathbf{C}$ is called additive, if it satisfies $A(x y)=A(x)+$ $A(y)$ for all $x, y \in G$.

A character of $G$ is a homomorphism from $G$ into the multiplicative group of non-zero complex numbers.

By $N(G, \sigma)$ we mean the vector space of the solutions $\theta: G \rightarrow \mathbf{C}$ of the homogeneous equation

$$
\theta(x y)-\theta(\sigma(y) x)=0, \quad x, y \in G .
$$

If $G$ is a topological space, then we let $C(G)$ denote the algebra of all continuous functions from $G$ into $\mathbf{C}$.

If $G$ is a locally compact group, then we let $M_{C}(G)$ denote the space of all compactly supported, complex-valued Borel measures on $G$. For $\mu \in$ $M_{C}(G)$, we say that $\mu$ is $\sigma$-invariant if $\mu(f \circ \sigma)=\mu(f)$ for all $f \in C(G)$, i.e.,

$$
\int_{G} f(\sigma(t)) d \mu(t)=\int_{G} f(t) d \mu(t) \text { for all } f \in C(G) .
$$

\section{Solution of equation (1.4)}

In this section, we solve the integral-functional equation (1.4), i.e.,

$$
\int_{G}\{f(x y t)-f(\sigma(y) x t)\} d \mu(t)=f(x) g(y), \quad x, y \in G,
$$

by expressing its continuous solutions in terms of continuous characters.

The following theorem is an immediate consequence of Theorem 4.2 in [1]. It will be used in the proof of Theorem 3.3. For the notation $N(G, \sigma)$ see section 2 .

Theorem 3.1. Let $G$ be a group, let $\sigma$ be an involutive automorphism on $G$, and let $f, g, h: G \rightarrow \mathbf{C}$ be solutions of the functional equation

$$
f(x y)-f(\sigma(y) x)=2 g(x) h(y), \quad x, y \in G .
$$

Then we have the following possibilities: 
a) $g=0, h$ is arbitrary, $f \in N(G, \sigma)$.

b) $h=0, g$ is arbitrary, $f \in N(G, \sigma)$.

c) There exist a character $\chi$ of $G$ with $\chi \neq \chi \circ \sigma$, constants $\alpha, \beta \in \mathbf{C}$, $\gamma \in \mathbf{C} \backslash\{0\}$, and a function $\theta \in N(G, \sigma)$ such that

$$
\begin{aligned}
g & =\alpha \chi+\beta \chi \circ \sigma \\
h & =\gamma(\chi-\chi \circ \sigma), \\
f & =2 \gamma(\alpha \chi-\beta \chi \circ \sigma)+\theta
\end{aligned}
$$

d) There exist a character $\chi$ of $G$ with $\chi=\chi \circ \sigma$, constants $\alpha, \beta \in \mathbf{C}$, an additive function $A: G \rightarrow \mathbf{C}$ with $A \circ \sigma=-A$, and a function $\theta \in N(G, \sigma)$ such that

$$
\begin{aligned}
g & =\alpha \chi+\beta \chi A \\
h & =\chi A \\
f & =\alpha \chi A+\frac{1}{2} \beta \chi A^{2}+\theta .
\end{aligned}
$$

Conversely, the formulas above for $f, g$ and $h$ define solutions of (3.1). Moreover, if $G$ is a topological group, $g \neq 0, h \neq 0$, and $f, g, h \in C(G)$, then $\chi, \chi \circ \sigma, A, \theta \in C(G)$.

The following lemma will be used in the proof of Theorem 3.3 in which the integral-functional equation (1.4) will be solved.

Lemma 3.2 (3, Lemma 4.1). Let $G$ be a group and let $\sigma$ be an involutive automorphism on $G$. Let $\chi$ be a character of $G$ with $\chi \neq \chi \circ \sigma$, $A: G \rightarrow \mathbf{C}$ be an odd additive function, $\theta$ be a function in $N(G, \sigma)$, and $\alpha, \beta$ be complex numbers.

1. If $\alpha \chi+\beta \chi \circ \sigma+\theta=0$, then $\alpha=\beta=0$ and $\theta=0$.

2. If $A^{2}+\alpha A+\theta=0$, then $A=\theta=0$.

By help of Theorem 3.1 and Lemma 3.2, we now describe the complete continuous solution of (1.4). 
Theorem 3.3. Let $G$ be a locally compact group, let $\sigma$ be a continuous involutive automorphism on $G$, and let $\mu \in M_{C}(G)$. Assume that the pair $f, g \in C(G)$ is a solution of the functional equation (1.4). Then we have the following possibilities:

(a) $f=0, g$ is arbitrary in $C(G)$.

(b) $g=0, f \in\left\{k \in C(G) \mid x \mapsto \int_{G} k(x t) d \mu(t) \in N(G, \sigma)\right\}$.

(c) There exist a non-even continuous character $\chi$ of $G$ with $\mu(\chi) \neq 0$ and a non-zero complex number $\alpha$ such that

$$
f=\alpha \chi \quad \text { and } \quad g=\mu(\chi)(\chi-\chi \circ \sigma) .
$$

(d) There exist a continuous character $\chi$ of $G$ with $\mu(\chi) \neq 0, \mu(\chi \circ \sigma)=$ $-\mu(\chi)$, and non-zero complex numbers $\alpha, \beta$ such that

$$
f=\alpha \chi+\beta \chi \circ \sigma \quad \text { and } \quad g=\mu(\chi)(\chi-\chi \circ \sigma) .
$$

Conversely, the functions given with these properties satisfy the functional equation (1.4).

Proof. The first two cases are obvious, so we suppose that $f \neq 0$ and $g \neq 0$. Define $F: G \rightarrow \mathbf{C}$ by

$$
F(x)=2 \int_{G} f(x t) d \mu(t) \quad \text { for all } \quad x \in G .
$$

Since $\mu \in M_{C}(G)$ and $f \in C(G)$, we have $F \in C(G)$. Using (3.2), the equation (1.4) becomes

$$
F(x y)-F(\sigma(y) x)=2 f(x) g(y), \quad x, y \in G .
$$

Since $f, g \in C(G) \backslash\{0\}$, we know from Theorem 3.1 that there are only the following two cases:

Case 1: There exist a continuous character $\chi$ of $G$ with $\chi \neq \chi \circ \sigma$, constants $\alpha, \beta \in \mathbf{C}, \gamma \in \mathbf{C} \backslash\{0\}$, and a continuous function $\theta \in N(G, \sigma)$ such that

$$
\begin{aligned}
f & =\alpha \chi+\beta \chi \circ \sigma \\
g & =\gamma(\chi-\chi \circ \sigma) \\
F & =2 \gamma(\alpha \chi-\beta \chi \circ \sigma)+\theta
\end{aligned}
$$


Using the expression $F(x)=2 \int_{G} f(x t) d \mu(t)$ for all $x \in G$, we get that

$$
2 \gamma[\alpha \chi(x)-\beta \chi \circ \sigma(x)]+\theta(x)=2 \alpha \chi(x) \mu(\chi)+2 \beta \chi \circ \sigma(x) \mu(\chi \circ \sigma),
$$

for all $x \in G$. We reformulate the last equality as follows

$$
2 \alpha[\gamma-\mu(\chi)] \chi(x)-2 \beta[\gamma+\mu(\chi \circ \sigma)] \chi \circ \sigma(x)+\theta(x)=0,
$$

for all $x \in G$. According to Lemma 3.2(a), we obtain

$$
\left\{\begin{array}{l}
\alpha[\gamma-\mu(\chi)]=0 \\
\beta[\gamma+\mu(\chi \circ \sigma)]=0
\end{array}\right.
$$

Since $f \neq 0$, then at least one of $\alpha$ and $\beta$ is non-zero.

Subcase 1.1: Suppose that $\beta=0$. Then $\alpha \neq 0$ and hence $\gamma=\mu(\chi)$, which implies that $f=\alpha \chi$ and $g=\mu(\chi)(\chi-\chi \circ \sigma)$. This is case $(c)$ in our statement.

Subcase 1.2: Suppose that $\alpha=0$. Then $\beta \neq 0$ and hence $\gamma=-\mu(\chi \circ \sigma)$, which implies that $f=\beta \chi \circ \sigma$ and $g=-\mu(\chi \circ \sigma)(\chi-\chi \circ \sigma)=\mu(\chi \circ \sigma)(\chi \circ$ $\sigma-\chi)$. So we are in case $(c)$ with the continuous character $\chi \circ \sigma$ replacing $\chi$.

Subcase 1.3: We now suppose that $\alpha \neq 0$ and $\beta \neq 0$. Then $\gamma=\mu(\chi)=$ $-\mu(\chi \circ \sigma)$ which implies that $g=\mu(\chi)(\chi-\chi \circ \sigma)$. Then we arrive at the solution in case $(d)$. This completes case 1 .

Case 2: There exist a continuous character $\chi$ of $G$ with $\chi=\chi \circ \sigma$, constants $\alpha, \beta \in \mathbf{C}$, a continuous additive function $A: G \rightarrow \mathbf{C}$ with $A \circ \sigma=-A$, and a continuous function $\theta \in N(G, \sigma)$ such that

$$
\begin{aligned}
f & =\alpha \chi+\beta \chi A \\
g & =\chi A \\
F & =\alpha \chi A+\frac{1}{2} \beta \chi A^{2}+\theta
\end{aligned}
$$

A small computation based on (3.2) shows that

$$
\begin{aligned}
& \alpha \chi(x) A(x)+\frac{1}{2} \beta \chi(x) A^{2}(x)+\theta(x) \\
& \quad=2 \alpha \mu(\chi) \chi(x)+2 \beta \mu(\chi) A(x) \chi(x)+2 \beta \chi(x) \mu(A \chi),
\end{aligned}
$$


for all $x \in G$. We reformulate the last equation as follows

$$
\beta A^{2}+2[\alpha-2 \beta \mu(\chi)] A+\theta_{1}=0,
$$

where $\theta_{1}:=2\left(\frac{\theta}{\chi}\right)-4 \alpha \mu(\chi)-4 \beta \mu(A \chi)$. Since $\chi$ is even we have $\theta_{1} \in N(G, \sigma)$.

Subcase 2.1: Suppose that $\beta=0$. From (3.3) we see that $2 \alpha A+\theta_{1}=0$. Then $\alpha A \in N(G, \sigma)$, i.e., for all $x, y \in G$ we have

$$
\alpha A(x y)-\alpha A(\sigma(y) x)=0,
$$

which implies that $2 \alpha A(y)=0$ for all $y \in G$, i.e., $\alpha A=0$. Since $f=\alpha \chi$ and $f \neq 0$, then $\alpha \neq 0$ and hence $A=0$. Therefore $g=0$. This subcase does not apply, because $g \neq 0$ by assumption.

Subcase 2.2: We now suppose that $\beta \neq 0$. Dividing (3.3) by $\beta$ and using Lemma 3.2(b), we get that $A=0$. Hence $g=0$. Also this subcase does not apply, because $g \neq 0$. This finishes the necessity assertion.

Conversely, simple computations prove that the formulas above for $(f, g)$ define solutions of (1.4).

As a consequence of Theorem 3.3 one can obtain the following corollaries.

Corollary 3.4. Let $G$ be a locally compact group, let $\sigma$ be a continuous involutive automorphism on $G$, and let $\mu$ be a $\sigma$-invariant measure in $M_{C}(G)$. Then a pair $f, g \in C(G) \backslash\{0\}$ satisfies the functional equation (1.4) if and only if there exist a non-even continuous character $\chi$ of $G$ with $\mu(\chi) \neq 0$ and a non-zero complex number $\alpha$ such that

$$
f=\alpha \chi \quad \text { and } \quad g=\mu(\chi)(\chi-\chi \circ \sigma) \text {. }
$$

Proof. Let $\chi$ be a continuous character of $G$ such that $\mu(\chi) \neq 0$. Since $\mu$ is $\sigma$-invariant we have $\mu(\chi \circ \sigma)=\mu(\chi)$. This implies that $\mu(\chi \circ \sigma) \neq$ $-\mu(\chi)$. Indeed, $\mu(\chi \circ \sigma)=-\mu(\chi)$ would entail $\mu(\chi)=0$, contradicting our assumption. So a pair $f, g \in C(G) \backslash\{0\}$ satisfies the equation (1.4) if and only if it has the form stated in case $(c)$ of Theorem 3.3.

Corollary 3.5. Let $G$ be a locally compact group, let $\sigma$ be a continuous involutive automorphism on $G$, and let $\mu \in M_{C}(G)$. Then a function $f \in$ $C(G) \backslash\{0\}$ satisfies the functional equation (1.4) if and only if there exists a continuous character $\chi$ of $G$ with $\mu(\chi) \neq 0$ and $\mu(\chi \circ \sigma)=-\mu(\chi)$ such that

$$
f=-\mu(\chi)(\chi-\chi \circ \sigma)
$$


Proof. The proof follows on putting $g=-f$ in Theorem 3.3.

In the following corollaries let $G$ be a group, $n \in \mathbf{N}, z_{0}, z_{1}, \ldots, z_{n} \in G$ be arbitrarily fixed elements, and let $\sigma$ be an involutive automorphism on $G$. To illustrate our theory, we continue by discussing the solutions of Eq. (1.4), but now for the case of $\mu$ being supported by a finite set. We can of course get all solutions, continuous or not, by considering the special instance of the discrete topology on $G$.

Corollary 3.6. The non-zero functions $f, g: G \rightarrow \mathbf{C}$ satisfying the functional equation

$$
f\left(x y z_{0}\right)-f\left(\sigma(y) x z_{0}\right)=2 f(x) g(y), \quad x, y \in G,
$$

are the ones of the forms:

(a)

$$
f=\alpha \chi \quad \text { and } \quad g=\frac{\chi\left(z_{0}\right)}{2}(\chi-\chi \circ \sigma),
$$

where $\alpha$ is a non-zero complex number and $\chi$ is a non-even character of $G$.

(b)

$$
f=\alpha \chi+\beta \chi \circ \sigma \quad \text { and } \quad g=\frac{\chi\left(z_{0}\right)}{2}(\chi-\chi \circ \sigma),
$$

$\alpha, \beta$ are non-zero complex numbers and $\chi$ is a character of $G$ such that $\chi \circ \sigma\left(z_{0}\right)=-\chi\left(z_{0}\right)$.

Proof. The proof follows on putting $\mu=\frac{1}{2} \delta_{z_{0}}$ in Theorem 3.3.

As a consequence of Corollary 3.5 (or Corollary 3.6) we have the following result which is a natural extension of Van Vleck's equation (1.1).

Corollary 3.7. The non-zero solutions $f: G \rightarrow \mathbf{C}$ of the functional equation

$$
f\left(\sigma(y) x z_{0}\right)-f\left(x y z_{0}\right)=2 f(x) f(y), \quad x, y \in G,
$$

are the functions of the form $f=-\frac{\chi\left(z_{0}\right)}{2}(\chi-\chi \circ \sigma)$, where $\chi$ is a character of $G$ such that $\chi \circ \sigma\left(z_{0}\right)=-\chi\left(z_{0}\right)$. 
Proof. The proof follows on putting $\mu=\frac{1}{2} \delta_{z_{0}}$ in Corollary 3.5.

Corollary 3.8. The non-zero functions $f, g: G \rightarrow \mathbf{C}$ satisfying the functional equation

$$
\sum_{i=0}^{n}\left\{f\left(x y z_{i}\right)-f\left(\sigma(y) x z_{i}\right)\right\}=2 f(x) g(y), \quad x, y \in G,
$$

are the ones of the forms:

(a)

$$
f=\alpha \chi \quad \text { and } g=\sum_{i=0}^{n} \frac{\chi\left(z_{i}\right)}{2}(\chi-\chi \circ \sigma),
$$

where $\alpha$ is a non-zero complex number and $\chi$ is a non-even character of $G$ such that $\sum_{i=0}^{n} \chi\left(z_{i}\right) \neq 0$.

(b)

$$
f=\alpha \chi+\beta \chi \circ \sigma \quad \text { and } \quad g=\sum_{i=0}^{n} \frac{\chi\left(z_{i}\right)}{2}(\chi-\chi \circ \sigma)
$$

where $\alpha, \beta$ are non-zero complex numbers and $\chi$ is a character of $G$ such that $\sum_{i=0}^{n} \chi\left(z_{i}\right) \neq 0$ and

$$
\sum_{i=0}^{n} \chi \circ \sigma\left(z_{i}\right)=-\sum_{i=0}^{n} \chi\left(z_{i}\right)
$$

Proof. The proof follows on putting $\mu=\frac{1}{2} \sum_{i=0}^{n} \delta_{z_{i}}$ in Theorem 3.3.

In view of Corollary 3.8, we have the following.

Corollary 3.9. The non-zero solutions $f: G \rightarrow \mathbf{C}$ of the functional equation

$$
\sum_{i=0}^{n}\left\{f\left(\sigma(y) x z_{i}\right)-f\left(x y z_{i}\right)\right\}=2 f(x) f(y), \quad x, y \in G,
$$

are the functions of the form

$$
f=-\sum_{i=0}^{n} \frac{\chi\left(z_{i}\right)}{2}(\chi-\chi \circ \sigma)
$$

where $\chi$ is a character of $G$ such that $\sum_{i=0}^{n} \chi\left(z_{i}\right) \neq 0$ and $\sum_{i=0}^{n} \chi \circ \sigma\left(z_{i}\right)=$ $-\sum_{i=0}^{n} \chi\left(z_{i}\right)$. 
Proof. The proof follows on putting $g=-f$ in Corollary 3.8.

\section{Solution of equation (1.5)}

In this section, we solve the integral-functional equation (1.5), namely

$$
\int_{G}\{f(x y t)-f(\sigma(y) x t)\} d \mu(t)=g(x) f(y), \quad x, y \in G,
$$

by expressing its continuous solutions in terms of continuous characters and continuous additive functions.

Lemma 4.1. Let $G$ be a locally compact group, let $\sigma$ be a continuous involutive automorphism on $G$, and let $\mu \in M_{C}(G)$. The following pairs of functions $f, g: G \rightarrow \mathbf{C}$ are continuous solutions of the functional equation (1.5):

(a)

$$
f=c(\chi-\chi \circ \sigma) \text { and } g=\mu(\chi) \chi+\mu(\chi \circ \sigma) \chi \circ \sigma,
$$

where $c \in \mathbf{C}$ and $\chi$ is a continuous character of $G$, and

(b)

$$
f=\chi A \text { and } g=2 \mu(\chi) \chi,
$$

where $\chi$ is a continuous character of $G$ such that $\chi \circ \sigma=\chi$ and where $A$ is a continuous additive function on $G$ such that $A \circ \sigma=-A$.

Proof. (a) Assume that there exist a continuous character $\chi$ of $G$ and a complex number $c \in \mathbf{C}$ such that $f=c(\chi-\chi \circ \sigma)$ and $g=\mu(\chi) \chi+\mu(\chi \circ$ $\sigma) \chi \circ \sigma$. Then $f, g \in C(G)$ and a small computation shows that

$$
f(x y t)-f(\sigma(y) x t)=c[\chi(x) \chi(t)+\chi \circ \sigma(x) \chi \circ \sigma(t)][\chi(y)-\chi \circ \sigma(y)],
$$

for all $x, y, t \in G$. Using (4.1) at the first equality sign: 


$$
\begin{aligned}
& \int_{G}\{f(x y t)-f(\sigma(y) x t)\} d \mu(t) \\
& \quad=c[\chi(y)-\chi \circ \sigma(y)] \int_{G}\{\chi(x) \chi(t)+\chi \circ \sigma(x) \chi \circ \sigma(t)\} d \mu(t) \\
& =c[\chi(y)-\chi \circ \sigma(y)][\mu(\chi) \chi(x)+\mu(\chi \circ \sigma) \chi \circ \sigma(x)] \\
& \quad=g(x) f(y) .
\end{aligned}
$$

So the pair $(f, g)$ is a solution of (1.5).

(b) Assume that there exist a continuous character $\chi$ of $G$ with $\chi \circ \sigma=\chi$ and a continuous additive function $A$ on $G$ with $A \circ \sigma=-A$ such that $f=\chi A$ and $g=2 \mu(\chi) \chi$. Then $f, g \in C(G)$ and a small computation shows that

$$
f(x y t)-f(\sigma(y) x t)=2 \chi(x) \chi(y) A(y) \chi(t), \quad \text { for all } \quad x, y, t \in G .
$$

Using (4.2) at the first equality sign:

$$
\begin{aligned}
\int_{G}\{f(x y t)-f(\sigma(y) x t)\} d \mu(t) & =2 \chi(x) \chi(y) A(y) \int_{G} \chi(t) d \mu(t) \\
& =2 \chi(x) \chi(y) A(y) \mu(\chi) \\
& =f(x) g(y) .
\end{aligned}
$$

So the pair $(f, g)$ is a solution of (1.5).

The second main theorem of the present paper asserts that the converse result to Lemma 4.1 is also valid. It reads as follows:

Theorem 4.2. Let $G$ be a locally compact group, let $\sigma$ be a continuous involutive automorphism on $G$, and let $\mu \in M_{C}(G)$. Let the pair $f, g \in$ $C(G) \backslash\{0\}$ be a solution of the functional equation (1.5). Then there exists a continuous character $\chi$ of $G$ such that

$$
g=\mu(\chi) \chi+\mu(\chi \circ \sigma) \chi \circ \sigma .
$$

Furthermore, we have the following possibilities:

(a) If $\chi \neq \chi \circ \sigma$, then there exists a constant $c \in \mathbf{C} \backslash\{0\}$ such that

$$
f=c(\chi-\chi \circ \sigma)
$$


(b) If $\chi=\chi \circ \sigma$, then there exists a continuous additive function $A: G \rightarrow$ $\mathbf{C}$ with $A \circ \sigma=-A$ such that

$$
f=\chi A \text {. }
$$

Conversely, the formulas above for $g$ and $f$ define continuous solutions of (1.5).

Proof. It remains to prove the necessity assertion. Similarly to the proof of Theorem 3.3 we define $F: G \rightarrow \mathbf{C}$ by

$$
F(x)=2 \int_{G} f(x t) d \mu(t) \quad \text { for all } \quad x \in G .
$$

So $F \in C(G)$ and the equation (1.5) becomes

$$
F(x y)-F(\sigma(y) x)=2 g(x) f(y), \quad x, y \in G .
$$

Since $g, f \in C(G) \backslash\{0\}$, we know from Theorem 3.1 that there are only the following two cases:

Case 1: There exist a continuous character $\chi$ of $G$ with $\chi \neq \chi \circ \sigma$, constants $\alpha, \beta \in \mathbf{C}, \gamma \in \mathbf{C} \backslash\{0\}$, and a continuous function $\theta \in N(G, \sigma)$ such that

$$
\begin{aligned}
g & =\alpha \chi+\beta \chi \circ \sigma \\
f & =\gamma(\chi-\chi \circ \sigma) \\
F & =2 \gamma(\alpha \chi-\beta \chi \circ \sigma)+\theta
\end{aligned}
$$

Since $F(x)=2 \int_{G} f(x t) d \mu(t)$ for all $x \in G$, we have

$$
2 \gamma[\alpha \chi(x)-\beta \chi \circ \sigma(x)]+\theta(x)=2 \gamma[\chi(x) \mu(\chi)-\chi \circ \sigma(x) \mu(\chi \circ \sigma)],
$$

for all $x \in G$. Since $\gamma \neq 0$, we can write the last equation as follows

$$
[\alpha-\mu(\chi)] \chi(x)+[\mu(\chi \circ \sigma)-\beta] \chi \circ \sigma(x)+\theta(x)=0,
$$

for all $x \in G$. Using Lemma 3.2(a), we get that $\alpha=\mu(\chi)$ and $\beta=\mu(\chi \circ \sigma)$. From this, we see that $g=\mu(\chi) \chi+\mu(\chi \circ \sigma) \chi \circ \sigma$ and arrive at the solution in case $(a)$ with $c=\gamma$. 
Case 2: There exist a continuous character $\chi$ of $G$ with $\chi=\chi \circ \sigma$, constants $\alpha, \beta \in \mathbf{C}$, a continuous additive function $A: G \rightarrow \mathbf{C}$ with $A \circ \sigma=-A$, and a continuous function $\theta \in N(G, \sigma)$ such that

$$
\begin{aligned}
g & =\alpha \chi+\beta \chi A, \\
f & =\chi A, \\
F & =\alpha \chi A+\frac{1}{2} \beta \chi A^{2}+\theta .
\end{aligned}
$$

Since $F(x)=2 \int_{G} f(x t) d \mu(t)$ for all $x \in G$, then a small computation shows that

$$
\alpha \chi(x) A(x)+\frac{1}{2} \beta \chi(x) A^{2}(x)+\theta(x)=2 \mu(\chi) \chi(x) A(x)+2 \chi(x) \mu(A \chi),
$$

for all $x \in G$. We reformulate the last equation as follows

$$
\beta A^{2}+2[\alpha-2 \mu(\chi)] A+\theta_{1}=0,
$$

where $\theta_{1}:=2\left(\frac{\theta}{\chi}\right)-4 \mu(A \chi)$. Since $\chi$ is even we have $\theta_{1} \in N(G, \sigma)$.

Subcase 2.1: Suppose that $\beta=0$. Hence $[\alpha-2 \mu(\chi)] A \in N(G, \sigma)$, which implies that $[\alpha-2 \mu(\chi)] A=0$. Since $f \neq 0$ we have $A \neq 0$, and hence $\alpha-2 \mu(\chi)=0$, i.e., $\alpha=2 \mu(\chi)$. Therefore $g=2 \mu(\chi) \chi$ and we arrive at the solution in case $(b)$.

Subcase 2.2: We now suppose that $\beta \neq 0$. According to Lemma 3.2(b), we get that $A=0$. Hence $f=0$. This subcase does not apply, because $f \neq 0$ by assumption. This finishes the proof.

As a consequence of Theorem 4.2, we have the following result on the solution of the functional equation

$$
f\left(x y z_{0}\right)-f\left(\sigma(y) x z_{0}\right)=2 g(x) f(y), \quad x, y \in G,
$$

which contains the solution of Van Vleck's equation on abelian groups.

Corollary 4.3. Let $G$ be a group, $z_{0} \in G$ be a fixed element, and $\sigma$ be an involutive automorphism on $G$. Let $f, g: G \rightarrow \mathbf{C}$ be non-zero functions satisfying the functional equation (4.3). Then there exists a character $\chi$ of $G$ such that

$$
g=\frac{\chi\left(z_{0}\right)}{2} \chi+\frac{\chi \circ \sigma\left(z_{0}\right)}{2} \chi \circ \sigma
$$

Furthermore, we have the following possibilities: 
(a) If $\chi \neq \chi \circ \sigma$, then there exists a constant $c \in \mathbf{C} \backslash\{0\}$ such that

$$
f=c(\chi-\chi \circ \sigma)
$$

(b) If $\chi=\chi \circ \sigma$, then there exists an additive function $A: G \rightarrow \mathbf{C}$ with $A \circ \sigma=-A$ such that

$$
f=\chi A \text {. }
$$

Conversely, the formulas above for $g$ and $f$ define solutions of (4.3).

Proof. The proof follows on putting $\mu=\frac{1}{2} \delta_{z_{0}}$ in Theorem 4.2.

As another consequence of Theorem 4.2, we have the following result on the solution of the functional equation

$$
\sum_{i=0}^{n}\left\{f\left(x y z_{i}\right)-f\left(\sigma(y) x z_{i}\right)\right\}=2 g(x) f(y), \quad x, y \in G
$$

which generalizes the equation (4.3).

Corollary 4.4. Let $G$ be a group, $z_{0}, z_{1}, \ldots, z_{n} \in G$ be fixed elements, and $\sigma$ be an involutive automorphism on $G$. Let $f, g: G \rightarrow \mathbf{C}$ be nonzero functions satisfying the functional equation (4.4). Then there exists a character $\chi$ of $G$ such that

$$
g=\sum_{i=0}^{n}\left[\frac{\chi\left(z_{i}\right)}{2} \chi+\frac{\chi \circ \sigma\left(z_{i}\right)}{2} \chi \circ \sigma\right]
$$

Furthermore, we have the following possibilities:

(a) If $\chi \neq \chi \circ \sigma$, then there exists a constant $c \in \mathbf{C} \backslash\{0\}$ such that

$$
f=c(\chi-\chi \circ \sigma)
$$

(b) If $\chi=\chi \circ \sigma$, then there exists an additive function $A: G \rightarrow \mathbf{C}$ with $A \circ \sigma=-A$ such that

$$
f=\chi A \text {. }
$$

Conversely, the formulas above for $g$ and $f$ define solutions of (4.4). 
Proof. The proof follows on putting $\mu=\frac{1}{2} \sum_{i=0}^{n} \delta_{z_{i}}$ in Theorem 4.2.

\section{Examples}

Example 5.1. Let $G=(\mathbf{R},+), \sigma(x)=-x$ for all $x \in \mathbf{R}, z_{0} \in \mathbf{R} \backslash\{0\}$ be a fixed element, and let $\mu=\frac{1}{2} \delta_{z_{0}}$.

We indicate here the corresponding continuous solutions of Eqs. (1.4), (1.5) and (1.6) by the help of Theorems 3.3 and 4.2 and Corollary 3.5.

The continuous characters on $\mathbf{R}$ are known to be $\chi(x)=e^{\lambda x}, x \in \mathbf{R}$, where $\lambda$ ranges over $\mathbf{C}$ (see for instance [6, Example 3.7(a)]). The condition $\mu(\chi \circ \sigma)=-\mu(\chi)$, i.e. $\chi\left(2 z_{0}\right)=-1$, of Theorem $3.3(d)$ becomes $e^{2 \lambda z_{0}}=-1$, which reduces to $\lambda=i \frac{(2 n+1) \pi}{2 z_{0}}$, where $n \in \mathbf{Z}$. The relevant characters are thus

$$
\chi_{n}(x):=e^{i \frac{(2 n+1) \pi}{2 z_{0}} x}, \quad x \in \mathbf{R}, \text { and } n \in \mathbf{Z} .
$$

The continuous additive functions on $\mathbf{R}$ are the functions of the form $A(x)=\alpha x, x \in \mathbf{R}$, where the constant $\alpha$ ranges over $\mathbf{C}$ (see for instance [6, Corollary 2.4]).

That $\theta \in N(\mathbf{R}, \sigma)$ is equivalent to $\theta(x+y)=\theta(x-y)$ for all $x, y \in \mathbf{R}$, i.e., $\theta(x)=\theta(x+2 y)$ for all $x, y \in \mathbf{R}$. Since $\mathbf{R}$ is 2-divisible, then each function in $N(\mathbf{R}, \sigma)$ is a constant. From this we infer that a function $f \in\left\{k \in C(\mathbf{R}) \mid x \mapsto \frac{1}{2} k\left(x+z_{0}\right) \in N(\mathbf{R}, \sigma)\right\}$ if and only if $f$ is a constant function.

In conclusion, by help of Theorem 3.3 we find that the continuous solutions $f, g: \mathbf{R} \rightarrow \mathbf{C}$ of the functional equation (1.4), which is here

$$
f\left(x+y+z_{0}\right)-f\left(x-y+z_{0}\right)=2 f(x) g(x), \quad x, y \in \mathbf{R},
$$

are

(a) $f=0$ and $g$ is arbitrary in $C(\mathbf{R})$.

(b) $f$ is a non-zero constant and $g=0$.

(c) $f(x)=\alpha e^{\lambda x}$ and

$$
g(x)=\frac{e^{\lambda\left(x+z_{0}\right)}-e^{-\lambda\left(x-z_{0}\right)}}{2}, \quad x \in \mathbf{R},
$$

for some non-zero complex numbers $\alpha, \lambda$. 
The generalized Van Vleck's equation on locally compact groups 561

(d)

$$
\begin{aligned}
f(x) & =\alpha e^{i \frac{(2 n+1) \pi}{2 z_{0}} x}+\beta e^{-i \frac{(2 n+1) \pi}{2 z_{0}} x} \\
& =(\alpha+\beta) \cos \left(\frac{(2 n+1) \pi}{2 z_{0}} x\right)+i(\alpha-\beta) \sin \left(\frac{(2 n+1) \pi}{2 z_{0}} x\right),
\end{aligned}
$$

and

$$
g(x)=(-1)^{n+1} \sin \left(\frac{(2 n+1) \pi}{2 z_{0}} x\right), \quad x \in \mathbf{R},
$$

for some $\alpha, \beta \in \mathbf{C} \backslash\{0\}$ and $n \in \mathbf{Z}$.

Now we seek the solutions $f, g \in C(\mathbf{R}) \backslash\{0\}$ of the functional equation (1.5) which is here

$$
f\left(x+y+z_{0}\right)-f\left(x-y+z_{0}\right)=2 g(x) f(x), \quad x, y \in \mathbf{R} .
$$

According to Theorem 4.2, we see that there exists a constant $\lambda \in \mathbf{C}$ such that

$$
g(x)=\frac{e^{i \lambda\left(x+z_{0}\right)}+e^{-i \lambda\left(x+z_{0}\right)}}{2}=\cos \left(\lambda\left(x+z_{0}\right)\right), \quad x \in \mathbf{R} .
$$

Furthermore, we have the following possibilities:

(a) If $\lambda \neq 0$, then there exists $\alpha \in \mathbf{C} \backslash\{0\}$ such that

$$
f(x)=\alpha \frac{e^{i \lambda x}-e^{-i \lambda x}}{2 i}=\alpha \sin (\lambda x), \quad x \in \mathbf{R} .
$$

(b) If $\lambda=0$, then $g=1$ and there exists $\alpha \in \mathbf{C} \backslash\{0\}$ such that

$$
f(x)=\alpha x, \quad x \in \mathbf{R} .
$$

Finally, by help of Corollary 3.5 we see that the solutions $f \in C(\mathbf{R}) \backslash\{0\}$ of the functional equation (1.6), which is here Van Vleck's equation (1.1), are the sine functions

$$
f(x)=(-1)^{n} \sin \left(\frac{(2 n+1) \pi}{2 z_{0}} x\right), \quad x \in \mathbf{R}, \quad n \in \mathbf{Z} .
$$

This result can be found e.g. in [7]. 
Example 5.2. For an application of our results on a non-abelian group, consider the 3-dimensional Heisenberg group $G=H_{3}$ described in [6, Example $A .17(a)$ ], and take as the involutive automorphism

$$
\begin{gathered}
\sigma\left(\begin{array}{lll}
1 & a & c \\
0 & 1 & b \\
0 & 0 & 1
\end{array}\right)=\left(\begin{array}{lll}
1 & b & c \\
0 & 1 & a \\
0 & 0 & 1
\end{array}\right)^{-1}=\left(\begin{array}{ccc}
1 & -b & -c+a b \\
0 & 1 & -a \\
0 & 0 & 1
\end{array}\right) \text { for } a, b, c \in \mathbf{R} . \\
\text { Let } Z_{0}=\left(\begin{array}{ccc}
1 & a_{0} & c_{0} \\
0 & 1 & b_{0} \\
0 & 0 & 1
\end{array}\right) \text { be a fixed element of } H_{3} \text { and let } \mu=\frac{1}{2} \delta_{Z_{0}} .
\end{gathered}
$$

We indicate here the corresponding continuous solutions of Eqs. (1.4), (1.5) and (1.6).

The continuous characters on $H_{3}$ are parametrized by $(\alpha, \beta) \in \mathbf{C}^{2}$ as follows (see, e.g., [1, Example 5.2]).

$$
\chi_{\alpha, \beta}\left(\begin{array}{ccc}
1 & a & c \\
0 & 1 & b \\
0 & 0 & 1
\end{array}\right)=e^{\alpha a+\beta b} \quad \text { for } a, b, c \in \mathbf{R} .
$$

We compute that $\chi_{\alpha, \beta} \circ \sigma=\chi_{-\beta,-\alpha}$, so $\chi_{\alpha, \beta} \circ \sigma=\chi_{\alpha, \beta}$, if and only if $\beta=-\alpha$, and in that case

$$
\chi_{\alpha,-\alpha}\left(\begin{array}{ccc}
1 & a & c \\
0 & 1 & b \\
0 & 0 & 1
\end{array}\right)=e^{\alpha(a-b)} \quad \text { for } a, b, c \in \mathbf{R} .
$$

In view of [1, Example 5.2], the continuous odd additive functions on $H_{3}$ are parametrized by $\gamma \in \mathbf{C}$ as follows

$$
A_{\gamma}\left(\begin{array}{ccc}
1 & a & c \\
0 & 1 & b \\
0 & 0 & 1
\end{array}\right)=\gamma(a+b) \text { for } a, b, c \in \mathbf{R}
$$

Now we are in the position to describe the solutions $f, g \in C\left(H_{3}\right) \backslash\{0\}$ of the functional equation (1.5), which is here

$$
f\left(X Y Z_{0}\right)-f\left(\sigma(Y) X Z_{0}\right)=2 g(X) f(Y), \quad X, Y \in H_{3} .
$$

By help of Theorem 4.2 we see that there exist constants $\alpha, \beta \in \mathbf{C}$ such that

$$
g\left(\begin{array}{ccc}
1 & a & c \\
0 & 1 & b \\
0 & 0 & 1
\end{array}\right)=\frac{e^{\alpha\left(a+a_{0}\right)+\beta\left(b+b_{0}\right)}+e^{-\beta\left(a+a_{0}\right)-\alpha\left(b+b_{0}\right)}}{2}, \quad a, b, c \in \mathbf{R} .
$$


Furthermore, we have the following possibilities:

(a) If $\beta \neq-\alpha$, then there exists $\gamma \in \mathbf{C} \backslash\{0\}$ such that

$$
f\left(\begin{array}{ccc}
1 & a & c \\
0 & 1 & b \\
0 & 0 & 1
\end{array}\right)=\gamma\left[e^{\alpha a+\beta b}-e^{-\beta a-\alpha b}\right], \quad a, b, c \in \mathbf{R}
$$

(b) If $\beta=-\alpha$, then $g\left(\begin{array}{lll}1 & a & c \\ 0 & 1 & b \\ 0 & 0 & 1\end{array}\right)=e^{\alpha\left(a-b+a_{0}-b_{0}\right)}$ and there exists $\gamma \in \mathbf{C} \backslash\{0\}$ such that

$$
f\left(\begin{array}{ccc}
1 & a & c \\
0 & 1 & b \\
0 & 0 & 1
\end{array}\right)=\gamma(a+b) e^{\alpha(a-b)}, \quad a, b, c \in \mathbf{R}
$$

Now we seek the solutions $f, g \in C\left(H_{3}\right) \backslash\{0\}$ of the functional equation (1.4) which is here

$$
f\left(X Y Z_{0}\right)-f\left(\sigma(Y) X Z_{0}\right)=2 f(X) g(Y), \quad X, Y \in H_{3} .
$$

The condition $\mu\left(\chi_{\alpha, \beta} \circ \sigma\right)=-\mu\left(\chi_{\alpha, \beta}\right)$, i.e. $\chi_{-\beta,-\alpha}\left(Z_{0}\right)=-\chi_{\alpha, \beta}\left(Z_{0}\right)$, of Theorem $3.3(d)$ becomes $e^{\left(a_{0}+b_{0}\right)(\alpha+\beta)}=-1$, which breaks the job into two cases: $b_{0}=-a_{0}$ or $b_{0} \neq-a_{0}$.

Case 1: Suppose that $b_{0}=-a_{0}$. A small computation shows that $\chi_{-\beta,-\alpha}\left(Z_{0}\right) \neq$ $-\chi_{\alpha, \beta}\left(Z_{0}\right)$, for all $\alpha, \beta \in \mathbf{C}$. Then, using Theorem 3.3, the solutions $f, g \in$ $C\left(H_{3}\right) \backslash\{0\}$ of Eq. (5.1) are the functions of the forms:

$$
\begin{aligned}
& f\left(\begin{array}{lll}
1 & a & c \\
0 & 1 & b \\
0 & 0 & 1
\end{array}\right)=\gamma e^{\alpha a+\beta b}, \\
& g\left(\begin{array}{lll}
1 & a & c \\
0 & 1 & b \\
0 & 0 & 1
\end{array}\right)=\frac{e^{\alpha\left(a+a_{0}\right)+\beta\left(b+b_{0}\right)}-e^{-\beta\left(a-b_{0}\right)-\alpha\left(b-a_{0}\right)}}{2}
\end{aligned}
$$

for all $a, b, c \in \mathbf{R}$, where $\alpha, \beta, \gamma$ are complex numbers such that $\beta \neq-\alpha$ and $\gamma \neq 0$. 
Case 2: Suppose that $b_{0} \neq-a_{0}$. A small computation shows that $\chi_{-\beta,-\alpha}\left(Z_{0}\right)=$ $-\chi_{\alpha, \beta}\left(Z_{0}\right)$ if and only if $\beta=i \frac{(2 n+1) \pi}{a_{0}+b_{0}}-\alpha$, where $n \in \mathbf{Z}$. Then, using Theorem 3.3, the solutions $f, g \in C\left(H_{3}\right) \backslash\{0\}$ of Eq. (5.1) are the ones of the forms:

(1)

$$
\begin{aligned}
f\left(\begin{array}{lll}
1 & a & c \\
0 & 1 & b \\
0 & 0 & 1
\end{array}\right) & =\gamma e^{\alpha a+\beta b} \\
g\left(\begin{array}{lll}
1 & a & c \\
0 & 1 & b \\
0 & 0 & 1
\end{array}\right) & =\frac{e^{\alpha\left(a+a_{0}\right)+\beta\left(b+b_{0}\right)}-e^{-\beta\left(a-b_{0}\right)-\alpha\left(b-a_{0}\right)}}{2}
\end{aligned}
$$

for all $a, b, c \in \mathbf{R}$, where $\alpha, \beta, \gamma$ are complex numbers such that $\beta \neq$ $-\alpha$ and $\gamma \neq 0$.

(2)

$$
\begin{aligned}
& f\left(\begin{array}{lll}
1 & a & c \\
0 & 1 & b \\
0 & 0 & 1
\end{array}\right)=e^{\alpha(a-b)}\left[\gamma e^{i \frac{(2 n+1) \pi}{a_{0}+b_{0}} b}+\delta e^{-i \frac{(2 n+1) \pi}{a_{0}+b_{0}} a}\right] \\
& g\left(\begin{array}{lll}
1 & a & c \\
0 & 1 & b \\
0 & 0 & 1
\end{array}\right)=e^{\alpha\left(a-b+a_{0}-b_{0}\right)+i \frac{(2 n+1) \pi}{a_{0}+b_{0}} b_{0}} \frac{e^{i \frac{(2 n+1) \pi}{a_{0}+b_{0}} b}-e^{-i \frac{(2 n+1) \pi}{a_{0}+b_{0}} a}}{2}
\end{aligned}
$$

for all $a, b, c \in \mathbf{R}$, where $\alpha \in \mathbf{C}, \gamma, \delta \in \mathbf{C} \backslash\{0\}$, and $n \in \mathbf{Z}$.

We complete this section by solving the functional equation (1.6), which is here

$$
f\left(\sigma(Y) X Z_{0}\right)-f\left(X Y Z_{0}\right)=2 f(X) f(Y), \quad X, Y \in H_{3} .
$$

By help of Corollary 3.5 and the previous discussion we see that we have the following two possibilities:

If $b_{0}=-a_{0}$, then the only solution of Eq. (5.2) is the function $f \equiv 0$. If $b_{0} \neq-a_{0}$, then the solutions $f \in C\left(H_{3}\right)$ of Eq. (5.2) are either $f \equiv 0$ or

$$
f\left(\begin{array}{lll}
1 & a & c \\
0 & 1 & b \\
0 & 0 & 1
\end{array}\right)=-e^{\alpha\left(a-b+a_{0}-b_{0}\right)+i \frac{(2 n+1) \pi}{a_{0}+b_{0}} b_{0}} \frac{e^{i \frac{(2 n+1) \pi}{a_{0}+b_{0}} b}-e^{-i \frac{(2 n+1) \pi}{a_{0}+b_{0}} a}}{2},
$$

for all $a, b, c \in \mathbf{R}$, where $\alpha \in \mathbf{C}$ and $n \in \mathbf{Z}$. 


\section{References}

[1] Ebanks, B. R., Stetkær, H.: d'Alembert's other functional equation on monoids with an involution. Aequationes Math. 89(1), pp. 187206, (2015).

[2] Fadli, B., Zeglami, D., Kabbaj, S.: A variant of Wilson's functional equation. Publ. Math. Debrecen 87(3-4), pp. 415-427, (2015).

[3] Fadli, B., Zeglami, D., Kabbaj, S.: A joint generalization of Van Vleck's and Kannappan's equations on groups. Adv. Pure Appl. Math. 6(3), pp. 179-188, (2015).

[4] Perkins, A.M., Sahoo, P.K.: On two functional equations with involution on groups related to sine and cosine functions. Aequationes Math. 89(5), pp. 1251-1263, (2015).

[5] Stetkær, H.: Functional equations on abelian groups with involution. Aequationes Math. 54(1-2), pp. 144-172, (1997).

[6] Stetkær, H.: Functional equations on groups. World Scientific Publishing Co, Singapore, (2013).

[7] Stetkær, H.: Van Vleck's functional equation for the sine. Aequationes Math. 90(1), pp. 25-34, (2016).

[8] Van Vleck, E.B.: A functional equation for the sine. Ann. Math. Second Ser. 11(4), pp. 161-165, (1910).

[9] Van Vleck, E.B.: A functional equation for the sine. Additional note. Ann. Math. 13(1/4) (1911-1912), 154, (...).

[10] Zeglami, D., Fadli, B., Kabbaj, S.: On a variant of $\mu$-Wilson's functional equation on a locally compact group. Aequationes Math. 89(5), pp. 1265-1280, (2015). 


\section{B. Fadli}

Department of Mathematics, Faculty of Sciences, IBN Tofail University, Bp: 14000. Kenitra, Morocco

e-mail : himfadli@gmail.com

\section{Zeglami}

Department of Mathematics, E.N.S.A.M, Moulay ISMAIL University, B.P. : 15290 Al Mansour-Meknes, Morocco

e-mail : zeglamidriss@yahoo.fr

and

\section{S. Kabbaj}

Department of Mathematics, Faculty of Sciences, IBN TOFAIL University, BP: 14000. Kenitra, MOROCCO

e-mail : samkabbaj@yahoo.fr 